\title{
AN ASYMPTOTIC FORMULA FOR POLYNOMIALS ORTHONORMAL WITH RESPECT TO A VARYING WEIGHT
}

\author{
A. V. KOMLOV AND S. P. SUETIN
}

\begin{abstract}
We obtain a strong asymptotic formula for the leading coefficient $\alpha_{n}(n)$ of a degree $n$ polynomial $q_{n}(z ; n)$ orthonormal on a system of intervals on the real line with respect to a varying weight. The weight depends on $n$ as $e^{-2 n Q(x)}$, where $Q(x)$ is a polynomial and corresponds to the "hard-edge case". The formula in Theorem 1 is quite similar to Widom's classical formula for a weight independent of $n$. In some sense, Widom's formulas are still true for a varying weight and are thus universal. As a consequence of the asymptotic formula we have that $\alpha_{n}(n) e^{-n w_{Q}}$ oscillates as $n \rightarrow \infty$ and, in a typical case, fills an interval (here $w_{Q}$ is the equilibrium constant in the external field $Q$ ).
\end{abstract}

\section{INTRODUCTION}

1.1. Let $Q(x)=x^{2 m}+\ldots$ be a monic real polynomial of even degree $2 m$; henceforth $Q$ will play the role of an "external field" (more precisely, a potential of an external field) in a standard potential-theoretic equilibrium problem.

We consider in the class $M_{1}(\mathbb{R})$ of unit (positive Borel) measures $\mu$ with support Supp $\mu$ on the real line $\mathbb{R}$, the following standard potential-theoretic equilibrium problem for the logarithmic potential $V^{\mu}(x)=-\int \log |x-t| d \mu(t)$ with external field $Q(x)$ (see [20. formulas (27), (28)], 38, [39, 21, as well as 29], 40], 12], 4]): find a measure $\lambda \in M_{1}(\mathbb{R})$ such that

$$
\begin{aligned}
V^{\lambda}(x)+Q(x) & \equiv w_{Q}, \quad x \in S, \quad S=\operatorname{Supp} \lambda, \\
\geq w_{Q}, & x \in \mathbb{R} \backslash S .
\end{aligned}
$$

There is a unique measure $\lambda=\lambda_{Q} \in M_{1}(\mathbb{R})$ satisfying (11); $\lambda$ is called the equilibrium measure and $w_{Q}$ is called the equilibrium constant. The equilibrium conditions (11) completely determine $\lambda$ (in the given class $M_{1}(\mathbb{R})$ ) and the remaining unknown parameters of the problem: the support $S=S(Q)$ of $\lambda$ and the equilibrium constant $w_{Q}$.

It is well-known (see [21], as well as 2.2 below) that the equilibrium measure $\lambda$ is a unique extremal measure, namely the (unique) solution of the following extremal problem:

$$
I_{Q}(\lambda)=\min _{\mu \in M_{1}(\mathbb{R})} I_{Q}(\mu),
$$

where the energy functional $I_{Q}$ is of the form

$$
I_{Q}(\mu):=\int_{\mathbb{R}} V^{\mu}(x) d \mu(x)+2 \int_{\mathbb{R}} Q(x) d \mu(x) .
$$

It is known (see [12, [13]) that for a polynomial potential $Q(x)$ the support $S$ of the equilibrium measure consists of several (non-intersecting) intervals, $S=\bigsqcup_{j=1}^{p} S_{j}$,

2010 Mathematics Subject Classification. Primary 42C05; Secondary 33C45, 33C50, 33D45.

Key words and phrases. Varying weight, orthonormal polynomials, strong asymptotics, equilibrium distributions. 
$S_{j}=\left[s_{2 j-1}, s_{2 j}\right]$, and the measure $\lambda$ is of the form

$$
d \lambda(x)=h_{1}(x) H_{+}^{1 / 2}(x) d x, \quad x \in S,
$$

where the function $h_{1}$ is holomorphic and, in the regular case, is different from zero on $S$ (and therefore on some neighborhood of $S$ ), $H(z)=H_{2 p}(z):=\prod_{j=1}^{2 p}\left(z-s_{j}\right)$, and the choice of the branch is such that $H^{1 / 2}(z) \sim z^{p}$ as $z \rightarrow \infty$; by $H_{+}^{1 / 2}(x)$ in (4) and everywhere else we understand the limit values of $H^{1 / 2}(z)$ as $z \rightarrow x \in S$ in the upper half-plane.

Let $P_{n}(x)=x^{n}+\ldots$ be a sequence of polynomials defined by the orthogonality conditions

$$
\int_{\mathbb{R}} P_{n}(x) x^{j} \varphi_{n}(x) d x=0, \quad j=0, \ldots, n-1,
$$

where $\varphi_{n}(x)>0$ is a given sequence of weight functions on $\mathbb{R}$ such that, for each $n$, each power $x^{k}$ is integrable with weight $\varphi_{n}$ on $\mathbb{R}$. The polynomial $P_{n}$ minimizes the norm

$$
\left(\int_{\mathbb{R}}\left|P_{n}(x)\right|^{2} \varphi_{n}(x) d x\right)^{1 / 2}
$$

in the class of all degree $n$ monic polynomials; the distribution of zeroes of such polynomials depends on the asymptotic behavior of the functions $\varphi_{n}$ as $n \rightarrow \infty$ (see [21], [22]). Let $p_{n}(x)=p_{n}(x ; n)=\gamma_{n}(n) x^{n}+\ldots, \gamma_{n}(n)>0$, be the corresponding sequence of orthonormal polynomials:

$$
\int_{\mathbb{R}}\left|p_{n}(x)\right|^{2} \varphi_{n}(x) d x=1, \quad n=1,2, \ldots
$$

Now let $\varphi_{n}(x)=\exp \{-2 n Q(x)\}$, where $Q(x)=x^{2 m}+\ldots$ is the above polynomial potential. Then [21]

$$
\left|p_{n}(z ; n)\right|^{1 / n} \rightarrow e^{w_{Q}-V^{\lambda}(z)}, \quad n \rightarrow \infty,
$$

locally uniformly (i.e., uniformly on compact subsets) in the domain $\overline{\mathbb{C}} \backslash S$. The relation (6) is usually referred to as a weak (or logarithmic) asymptotic formula for the polynomials $p_{n}(\cdot ; n)$; it implies a weak asymptotic formula for leading coefficients: $\gamma_{n}(n)^{1 / n} \rightarrow$ $e^{w_{Q}}, n \rightarrow \infty$.

In the theory of random matrices (see [34, [12, [35, [17, 33], 441, 26], [47, 66, [7, [16, [5]), the problem of finding strong asymptotic formulas for the polynomials $p_{n}(z ; n)$ and the coefficients $\gamma_{n}(n)$ arises naturally. In the "soft-edge case" such formulas were obtained in [13, formulas (1.63)-(1.65)]. In the present paper we obtain a strong asymptotic formula for the leading coefficient of the orthonormal polynomial in the "hardedge case". It turns out that formula (13) is similar to Widom's classical formula [48, $\S 6$, Theorem 6.2] for a weight independent of $n$ (see also [3, 42, 44]). For a single interval $E=[-1,1]$, the "hard-edge case" was studied in $[8$, Theorem 2]. The results of the present paper generalize the results of $[8$ to the case of an arbitrary number $p \geq 2$ of intervals.

Thus, the main goal of theis paper is to find, in the "hard-edge case", a strong asymptotic formula for the leading coefficients $\alpha_{n}(n)$ of the orthonormal polynomials $q_{n}(z ; n)$ (see (12)). We obtain such a formula in terms of Green's function $g(z, \infty)$ for the domain $D=\overline{\mathbb{C}} \backslash E$ and a divisor moving on the $g$-dimensional torus $\mathbb{T}^{g}$ according to the (generalized) Dubrovin equation (cf. [33, (A.2), (A.10)]. It turns out that this formula (13) is of the form of Widom's classical formula [48] in the case of polynomials orthogonal on several intervals with respect to a weight independent of $n$. It is therefore natural to call it Widom's formula for the case of varying weight. Notice that formula (13) immediately 
provides a qualitative description of the behavior of the leading coefficient $\alpha_{n}(n)$ : as $n \rightarrow \infty$, the quantity

$$
\alpha_{n}^{2}(n) \cdot e^{-2 n w_{Q}}
$$

oscillates near 1 as

$$
\exp \left\{-\sum_{j=1}^{g} g\left(\boldsymbol{z}_{j}(n), \infty\right)\right\}
$$

and in a "typical" case (see [25]) fills an interval (for details, see Corollary 4.2 below).

1.2. Let us recall the necessary definitions and notation.

Let $E=\bigsqcup_{j=1}^{p} E_{j}$, where $E_{j}=\left[e_{2 j-1}, e_{2 j}\right] \subset \operatorname{int} S_{j}, j=1, \ldots, p$ (int $S_{j}$ is the open interval corresponding to interval $S_{j}$ ), and $\lambda=\lambda_{Q, E} \in M_{1}(E)$ isR the (unique) equilibrium measure for the external field $Q$ with support on $E \subset \operatorname{int} S$ :

$$
V^{\lambda}(x)+Q(x)=w_{Q, E}, \quad x \in E .
$$

The measure $\lambda=\lambda_{Q, E}$ and only this measure is a solution (in the class $M_{1}(E)$ ) of the following extremal problem for the energy functional (3):

$$
I_{Q}(\lambda)=\min _{\nu \in M_{1}(E)} I_{Q}(\nu) .
$$

Notice that in (9), unlike (2), the minimum of the energy functional (3) is taken over the class of unit measures with supports in $E$; henceforth, we consider the equilibrium problem (8) only in the class $M_{1}(E)$ and, as a rule, we shall omit any mention of $E$. The equilibrium problem (8) corresponds to the limit case of the external field $Q \cdot\left(1 / \chi_{E}\right)$, where $\chi_{E}$ is the characteristic function of $E$. It is well-known that in this case the equilibrium measure is of the form (cf. (4))

$$
d \lambda(x)=\frac{h_{2}(x)}{H_{+}^{1 / 2}(x)} d x, \quad x \in E,
$$

where the function $h_{2}$ is holomorphic and is different from zero on $E$ :

$$
H(z)=H_{2 p}(z):=\prod_{j=1}^{2 p}\left(z-e_{j}\right), \quad H^{1 / 2}(z) \sim z^{p} \text { as } z \rightarrow \infty .
$$

For convenience, denote $H^{1 / 2}(z)$ by $W(z)$. Thus, $W^{2}=\prod_{j=1}^{2 p}\left(z-e_{j}\right)$ and $W(z) \sim z^{p}$ as $z \rightarrow \infty$.

Suppose we are given a weight

$$
\sigma_{n}(x)=\frac{i \rho_{n}(x)}{2 \pi W^{+}(x)}>0, \quad x \in E, \quad \rho_{n}(x):=p_{g}(x) e^{-2 n Q(x)},
$$

on $E$, where $p_{g}(x)=x^{g}+\ldots$ is a fixed polynomial of degree $g=p-1$ which has exactly one root in each gap (i.e., in the interval $\left.\left(e_{2 j}, e_{2 j+1}\right), j=1, \ldots, g\right)$ (thus, in each gap it changes sign exactly once; this polynomial is of auxiliary nature and is only needed to compensate the change of sign of $W^{+}(x) / i$ when passing from $E_{j}$ to $E_{j-1}$ ).

Let $q_{n}(x ; N)=\alpha_{n}(N) x^{n}+\ldots, \alpha_{n}(N)>0$, be polynomials orthonormal on $E$ with respect to $\sigma_{N}(x)$ :

$$
\int_{E} q_{n}(x ; N) q_{m}(x ; N) \sigma_{N}(x) d x=\delta_{n, m} .
$$

Recall once more that the main goal of this paper is to study the asymptotics of $\alpha_{n}(n)$, i.e., for $N=n$.

Let $\mathfrak{R}$ denote the hyperelliptic Riemann surface of genus $g=p-1$ with equation $W^{2}=\prod_{j=1}^{2 p}\left(z-e_{j}\right)$. We then have 
Theorem 1. Suppose the compact set $E=\bigsqcup_{j=1}^{p}\left[e_{2 j-1}, e_{2 j}\right] \subset \operatorname{int} S, \lambda=\lambda_{Q, E} \in M_{1}(E)$ is the equilibrium measure supported on $E$ for the problem (9) with external field $Q(x)=$ $x^{2 m}+\ldots$, and $w_{Q}$ the corresponding equilibrium constant. Then, for the leading coefficient $\alpha_{n}(n)$ of the polynomial $q_{n}(x ; n)$ orthonormal with respect to $\sigma_{n}(x)$, we have the following strong asymptotic formula:

$$
\alpha_{n}(n)=\text { const } \cdot e^{n w_{Q}} \cdot \exp \left\{-\frac{1}{2} \sum_{j=1}^{g} g\left(\boldsymbol{z}_{j}(n), \infty\right)\right\}(1+o(1)), \quad n \rightarrow \infty,
$$

where $o(1)=O\left(\delta^{n}\right), \delta \in(0,1)$, and const $=\operatorname{const}\left(E, p_{g}\right)$ depends only on the set $E$ and the auxiliary polynomial $p_{g}$. The points $\boldsymbol{z}_{j}(n)=\left(z_{j}(n), \pm\right)$ of the Riemann surface $\mathfrak{R}$ have the property that $z_{j}(n) \in\left[e_{2 j}, e_{2 j+1}\right], j=1, \ldots, g$, and are uniquely determined by a solution of the Jacobi inversion problem

$$
\sum_{j=1}^{g} \Omega_{k}\left(\boldsymbol{z}_{j}\right) \equiv-\sum_{j=1}^{g}\left\{n \lambda\left(E_{j}\right)\right\} B_{k j}+c_{k}, \quad k=1, \ldots, g,
$$

where $\{\cdot\}$ denotes the fractional part of the number, the $c_{k}$ are some constants (independent of $n)$, 三 denotes congruence modulo the periods of basis abelian differentials $d \Omega_{k}$ for $\Re, \Omega_{k}$ are the corresponding abelian integrals, and $\left\|B_{k j}\right\|$ is the Riemann matrix for $\Re$.

We remark that the multiplicative constant in (13) can be computed explicitly.

The proof of Theorem 1 is based on the following result. It turns out that, uniformly on compact subsets $K$ of the domain $D=\overline{\mathbb{C}} \backslash E$, the asymptotics of the orthonormal polynomials $q_{n}(z ; n)$ is completely determined in terms of the so-called Nuttall psi-function. A Nuttall psi-function $\Psi_{n}(\boldsymbol{z} ; \rho)$ is a function defined on the Riemann surface $\mathfrak{R}$ and which is a normalized solution of a Riemann boundary value problem with a multiplicative jump $\rho(x)$ defined on $E$ (see Problem 1 in 3.1 below). The next result generalizes the Ahiezer-Nuttall theorem (see [46, [1, [2, 32, 30]) to the case of polynomials orthogonal with respect to a varying weight (compare (16) with [44, $\S 2,1-3$, Theorem 1]).

Theorem 2. Let $\Psi_{n}(\boldsymbol{z})=\Psi_{n}\left(\boldsymbol{z} ; \rho_{n}\right)$ be a normalized solution of the boundary-value Problem 1 for $n \geq g, q_{n}(z ; n)$ a degree $n$ polynomial with a positive leading coefficient, orthonormal with respect to the varying weight

$$
\sigma_{n}(x)=\frac{i \rho_{n}(x)}{2 \pi W^{+}(x)}>0, \quad x \in E, \quad \rho_{n}(x)=p_{g}(x) e^{-2 n Q(x)} .
$$

Then we have a strong asymptotic formula:

$$
q_{n}(z ; n)=\Psi_{n}\left(z^{(1)}\right)(1+o(1)), \quad n \rightarrow \infty .
$$

In Theorem 2, the asymptotic formula (16) holds uniformly on any compact set $K$ in $\overline{\mathbb{C}}$ which lies outside the convex hull conv $E$ of the compact set $E$, with $o(1)=O\left(\delta^{n}\right)$, where $\delta \in(0,1)$. By $\Psi_{n}\left(z^{(1)}\right)$ we understand the values of the psi-function on the first sheet of $\mathfrak{R}$.

It follows directly from (16) that

$$
\alpha_{n}(n)=\varkappa_{n}(n)(1+o(1)), \quad n \rightarrow \infty,
$$

where $\varkappa_{n}(n)>0$ is the leading coefficient (i.e., the coefficient of $z^{n}$ ) of $\Psi_{n}\left(z^{(1)}\right)$ as $z \rightarrow \infty$. The strong asymptotic formula for $\varkappa_{n}(n)$ is established according to the general principles, as expounded in 44 for the case of the weight $\rho$ independent of $n$. It turns out that this formula looks like the right-hand side of (13). From this, one can then derive the desired strong asymptotic formula for the leading coefficient $\alpha_{n}(n)$.

The present paper is devoted to the proof of Theorem 1 under the assumption that Theorem 2 holds. A proof of Theorem 2 will be given in 24 . 
The results of this paper were partially announced in $[23]$.

\section{Auxiliary Results}

2.1. We need to recall some standard facts about hyperelliptic Riemann surfaces. Our notation and terminology mostly follow those of [49] (see also [45], 15]).

Consider the domain $D=D(E)=\overline{\mathbb{C}} \backslash E$, and let $g(z, \infty)=g_{D}(z, \infty)$ be Green's function for $D$ with singularity at the infinite point $z=\infty$. We have $g(z, \infty)=w_{0}-$ $V^{\gamma}(z)$, where $\gamma=\lambda_{0}$ is a Robin measure for the compact set $E$, and $w_{0}$ the Robin constant for $E$ (corresponding to the external field $Q \equiv 0)$ :

$$
V^{\gamma}(x) \equiv w_{0}, \quad x \in E, \quad \operatorname{Supp} \gamma=E .
$$

Let $\mathfrak{R}$ denote the hyperelliptic Riemann surface of genus $g=p-1 \geq 1$ given by the equation $W^{2}=\prod_{j=1}^{2 p}\left(z-e_{j}\right)$. It is a two-sheeted covering of the Riemann sphere $\overline{\mathbb{C}}$ ramified at the points $e_{j}$. To the intervals $E_{j}$ on $\overline{\mathbb{C}}$ there correspond closed cycles $\mathcal{L}_{j}$ on $\Re$ such that $\operatorname{proj} \mathcal{L}_{j}=E_{j}(\operatorname{proj}(\cdot)$ is the canonical projection of $\Re$ to $\overline{\mathbb{C}})$. If $\mathcal{L}=\mathcal{L}_{1} \sqcup \ldots \sqcup \mathcal{L}_{p}$, then $\mathfrak{R} \backslash \mathcal{L}$ breaks into two sheets, $D^{(1)}$ and $D^{(2)}$ (such that $\operatorname{proj}\left(D^{(l)}\right)=D$ ), i.e., $\mathfrak{R}=D^{(1)} \sqcup \mathcal{L} \sqcup D^{(2)}$. As a rule, we shall identify the first sheet $D^{(1)}$ with $D$.

The function $W(z)$ defined on $D$ as $W(z)=\sqrt{H(z)} \sim z^{p}, z \rightarrow \infty$, where $H(z)=$ $H_{2 p}(z):=\prod_{j=1}^{2 p}\left(z-e_{j}\right)$, extends to the second sheet by setting $W\left(z^{(2)}\right)=-\sqrt{H(z)} \sim$ $-z^{p}$ and becomes, on the Riemann surface $\mathfrak{R} \backslash\left\{\infty^{(1)}, \infty^{(2)}\right\}$, a holomorphic (singlevalued analytic) function. A point $\boldsymbol{z}$ on $\mathfrak{R}$ is determined by a pair $\boldsymbol{z}=(z, W)=$ $(z, \pm \sqrt{H(z)})=(z, \pm)$, and we have $z^{(1)}=(z,+)$ and $z^{(2)}=(z,-)$. Green's function harmonically extends to the second sheet of the Riemann surface by a change of sign: $g\left(z^{(2)}, \infty\right)=-g\left(z^{(1)}, \infty\right)$; thus $g\left(z^{(2)}, \infty\right)<0$ in $D^{(2)}$.

We choose an orientation for the curves $\mathcal{L}_{j}$ in such a way that when traversing $\mathcal{L}_{j}$ the domain $D^{(1)}$ is on the left, and $D^{(2)}$ is on the right. We now view the oriented curves $\mathcal{L}_{j}^{+}$, $j=1, \ldots, g$, as $a$-cycles $\boldsymbol{a}_{j}$ on $\mathfrak{R}$ and, by a standard procedure [45, 49], 15], [1] extend them by $b$-cycles $\boldsymbol{b}_{j}$ to a homology basis $\left\{\boldsymbol{a}_{j}, \boldsymbol{b}_{j}\right\}_{j=1, \ldots, g}$ on $\mathfrak{R}$. Let $d \Omega_{k}, k=1, \ldots, g$, be the corresponding $a$-normalized basis of holomorphic abelian differentials (1-forms): $\oint_{\boldsymbol{a}_{j}} d \Omega_{k}=\delta_{k j}, k, j=1, \ldots, g$. The matrix $\boldsymbol{B}=\left\|B_{k j}\right\|_{k, j=1, \ldots, g}$, where $B_{k j}=\oint_{\boldsymbol{b}_{j}} d \Omega_{k}$ are the $b$-periods of basis differentials, is a Riemann matrix: it is symmetric, $B_{k j}=B_{j k}$, and its imaginary part is positive definite, $\left\|\operatorname{Im} B_{j k}\right\|>0$. In our case, when $e_{j} \in \mathbb{R}$, all $B_{k j}$ are pure imaginary. The abelian integrals $\Omega_{k}(\boldsymbol{z})=\int_{e_{2 g+2}}^{\boldsymbol{z}} d \Omega_{k}$ are defined on $\mathfrak{R}$ uniquely modulo their $a$ - and $b$-periods. Since $\Omega_{k}^{\prime}(\boldsymbol{z})=s_{k}(z) / W(\boldsymbol{z})$, where $s_{k}(z)$ is a polynomial of degree $g-1$, the notation $d \Omega_{k}^{ \pm}(x)=s_{k}(x) d x / W^{ \pm}(x)$ makes sense for $x \in E$.

If a function $F(\boldsymbol{z})$ is defined on $\mathfrak{R} \backslash \mathcal{L}$ and $\boldsymbol{x} \in \mathcal{L}$, then the symbol $F^{(1)}(\boldsymbol{x})$ will denote the nontangent limit values when $D^{(1)} \ni \boldsymbol{z} \rightarrow \boldsymbol{x} \in \mathcal{L}$ whenever they exist. Similarly, we define $F^{(2)}(\boldsymbol{x})$ when $D^{(2)} \ni \boldsymbol{z} \rightarrow \boldsymbol{x} \in \mathcal{L}$.

Fix the standard basis $\boldsymbol{e}_{1}, \boldsymbol{e}_{2}, \ldots, \boldsymbol{e}_{g}$ in $\mathbb{R}^{g}:\left(\boldsymbol{e}_{k}\right)_{j}=\delta_{k j}$. Then the vectors $\boldsymbol{e}_{1}, \ldots, \boldsymbol{e}_{g}$, $\boldsymbol{B} \boldsymbol{e}_{1}, \ldots, \boldsymbol{B} \boldsymbol{e}_{g}$ are linearly independent over $\mathbb{R}$ and form a basis in $\mathbb{C}^{g}$. The quotient space $\mathbb{C}^{g} /\{\mathrm{N}+\boldsymbol{B M}\}$, where $\mathrm{N}, \mathrm{M} \in \mathbb{Z}^{g}$, is a $2 g$-dimensional real torus $\mathbb{T}^{2 g}$, called the Jacobian variety Jac $\mathfrak{R}$ of the Riemann surface $\mathfrak{R}$. Any vector $\boldsymbol{v} \in \mathbb{C}^{g}$ can be uniquely written in the form $\boldsymbol{v}=\boldsymbol{x}+\boldsymbol{B} \boldsymbol{y}+\mathrm{N}+\boldsymbol{B M}=\boldsymbol{x}+\boldsymbol{B} \boldsymbol{y}\left(\bmod\right.$ periods), $0 \leqslant(\boldsymbol{x})_{j},(\boldsymbol{y})_{j}<1$, $\mathrm{N}, \mathrm{M} \in \mathbb{Z}^{g}$. Sometimes, for brevity, we shall simply write $\boldsymbol{v} \equiv \boldsymbol{x}+\boldsymbol{B} \boldsymbol{y}$.

Unordered tuples $\left(\boldsymbol{z}_{1}, \ldots, \boldsymbol{z}_{g}\right), \boldsymbol{z}_{j} \in \mathfrak{R}$, form the $g$ th symmetric power $S^{g} \mathfrak{R}$ of $\mathfrak{R}$. Let $\boldsymbol{z}_{0}$ be some fixed point 1 of $\mathfrak{R}$. The vector function $A\left(\boldsymbol{z}_{1}, \ldots, \boldsymbol{z}_{g}\right)=\left(A_{1}, \ldots, A_{g}\right)$ with

\footnotetext{
${ }^{1}$ Henceforth, we shall always choose $\boldsymbol{z}_{0}=e_{2 g+2}$.
} 
coordinates

$$
A_{k}=A_{k}\left(\boldsymbol{z}_{1}, \ldots, \boldsymbol{z}_{g}\right) \equiv \sum_{j=1}^{g} \Omega_{k}\left(\boldsymbol{z}_{j}\right) \equiv\left\{\sum_{j=1}^{g} \int_{\boldsymbol{z}_{0}}^{\boldsymbol{z}_{j}}\right\} d \Omega_{k}, \quad k=1, \ldots, g,
$$

defines the Abel map $A: S^{g} \mathfrak{R} \rightarrow$ Jac $\Re$ (the integration paths in (19) are the same for all $k=1, \ldots, g)$. Given a vector $\boldsymbol{v}=\left(v_{1}, \ldots, v_{g}\right) \in \operatorname{Jac} \mathfrak{R}$ (more precisely, a class $[\boldsymbol{v}]$ of vectors equivalent to $\boldsymbol{v})$, the problem of determining an unordered tuple $\left(\boldsymbol{z}_{1}, \ldots, \boldsymbol{z}_{g}\right) \in$ $S^{g} \mathfrak{R}$ of points such that

$$
A_{k}\left(\boldsymbol{z}_{1}, \ldots, \boldsymbol{z}_{g}\right)=v_{k} \quad(\bmod \text { periods }), \quad k=1, \ldots, g,
$$

is called the Jacobi inversion problem. It is always solvable but not in general uniquely. We shall call an unordered tuple $\left(\boldsymbol{z}_{1}, \ldots, \boldsymbol{z}_{g}\right) \in S^{g} \mathfrak{R}$ of points a divisor and denote it by $d=\left(\boldsymbol{z}_{1}, \ldots, \boldsymbol{z}_{g}\right)$ or by $d=\boldsymbol{z}_{1}+\ldots+\boldsymbol{z}_{g}$.

Other standard results on hyperelliptic Riemann surfaces, which we will need here, can be found in [45], 49], [15, [1] (see also [44, Appendix A]).

2.2. For the sake of completeness, we want to mention some known results on the equilibrium distribution of a positive charge on a "conductor" in the presence of an "external field" (see the Gonchar-Rakhmanov theorem below). Here, we restrict ourselves to the case when the conductor is the real line $\mathbb{R}$ and the external field is given by a polynomial potential $Q(x)=x^{2 m}+\ldots \in \mathbb{R}[x]$. We remark that the notion of equilibrium in an external field first appeared in 1981-1982 in E. A. Rakhmanov's papers [38] and [39] dealing with the weak (logarithmic) asymptotics of polynomials orthogonal on the real line with respect to weights of the form $\exp \left\{-|x|^{\rho}\right\}, \rho>1$ (see also 20, 21, 29], 40]). The problems related to the limit behavior of extremal (in one sense or another) polynomials turned out to be important both for analysis and for applications. In the last two decades, these problems were shown to be closely related to problems on asymptotic properties of random matrices. For this reason, general methods for the study of the asymptotics of such polynomials are of great interest.

The next theorem was proved in 21 in the case of a sufficiently large external field (see also [10]). Here we state it in a rather special case, when the external field is given by a polynomial potential $Q$.

Henceforth, a measure is a positive Borel measure on the complex plane $\mathbb{C}$ with finite values on compact subsets of $\mathbb{C}, S_{\mu}=\operatorname{Supp} \mu$ is the support of $\mu,|\mu|=\mu(\mathbb{C})$, and $V^{\mu}$ is its logarithmic potential:

$$
V^{\mu}(z)=\int \log \frac{1}{|z-t|} d \mu(t), \quad z \in \mathbb{C} .
$$

Here, we only consider potentials for measures $\mu$ such that

$$
\int_{|t| \geq 1} \log \frac{1}{|t|} d \mu(t)>-\infty .
$$

For such measures $V^{\mu}(z) \in(-\infty,+\infty], z \in \mathbb{C}$. We remark that all the necessary notions and facts from potential theory can be found in [27] (see also [40]).

The set of all unit measures $\mu$ on $\mathbb{R}\left(S_{\mu} \subset \mathbb{R}\right)$ satisfying (22) will be denoted by $M_{1}(\mathbb{R})$. For measures $\mu \in M_{1}(\mathbb{R})$ we set

$$
\begin{gathered}
w(\mu ; Q):=\min _{x \in \mathbb{R}}\left(V^{\mu}+Q\right)(x), \\
I_{Q}(\mu):=\iint_{\mathbb{R} \times \mathbb{R}} \log \frac{1}{|x-y|} d \mu(x) d \mu(y)+2 \int_{\mathbb{R}} Q(x) d \mu(x) .
\end{gathered}
$$


Theorem (Gonchar-Rakhmanov (see [20], 21], 19])). Let $Q(x)=x^{2 m}+\ldots \in \mathbb{R}[x]$ be an arbitrary polynomial potential. There exists a unique (equilibrium) measure $\lambda=\lambda(Q)$ such that

$$
\left(V^{\lambda}+Q\right)(x) \equiv w(\lambda ; Q), \quad x \in S_{\lambda} .
$$

The measure $\lambda=\lambda(Q)$ is a unique solution of each of the following extremal problems:

(1) $\lambda$ maximizes $w(\mu ; Q)$ in the field $Q$ :

$$
w(\lambda ; Q)=\max _{\mu \in M_{1}(\mathbb{R})} w(\mu ; Q) ;
$$

(2) $\lambda$ maximizes the energy $I_{Q}(\mu)$ in the field $Q$ :

$$
I_{Q}(\lambda)=\min _{\mu \in M_{1}(\mathbb{R})} I_{Q}(\mu) .
$$

The equilibrium condition (25) can be equivalently written as

$$
\begin{aligned}
\left(V^{\lambda}+Q\right)(x) & =w, \quad x \in S_{\lambda}, \\
& \geq w, \quad x \in \mathbb{R} \backslash S_{\lambda},
\end{aligned}
$$

where $w$ is some constant; the first assertion of the theorem means that condition (28) uniquely determines the measure $\lambda \in M_{1}(\mathbb{R})$ (and thus the constant $w$ ). Condition (26) can be written as an implication:

$$
w(\mu ; Q) \geq w(\lambda ; Q),|\mu|=1 \Rightarrow \mu=\lambda .
$$

$\lambda=\lambda(Q)$ is called the equilibrium measure, corresponding to the potential $Q$, and $w=w(Q)=w(\lambda(Q) ; Q)$ is called the equilibrium constant. It follows directly from the equilibrium condition (25) that the potential $V^{\lambda}$ is continuous on $S_{\lambda}$ and is thus continuous on $\mathbb{C}$.

Setting

$$
S^{\lambda}=\left\{x \in \mathbb{R}:\left(V^{\lambda}+Q\right)(x)=w(\lambda ; Q)\right\},
$$

we can rewrite the equilibrium condition (28) in the following equivalent form:

$$
S_{\lambda} \subseteq S^{\lambda} .
$$

Notice that condition (31) is equivalent to the equilibrium condition (28): $\lambda$ is the only measure in $M_{1}(\mathbb{R})$ satisfying (31).

It is well-known that even for rather simple polynomials $Q$ the determination of the equilibrium measure $\lambda=\lambda(Q)$ and the extremal constant $w=w(Q)$ could be difficult because the support measure $\lambda$ is not known a priori. Calculations (based on the relations (28) and (29)) simplify when one knows that the support of the equilibrium measure is an interval (or several intervals; see 12, 13, 9]). This happens in the case considered here when the potential is a polynomial (see [13). Under the assumption that the support of the equilibrium measure $\lambda$ is an interval $[\alpha, \beta]$, we can find $\lambda$ and the potential $V^{\lambda}$ from the equilibrium condition (28), which leads to the well-studied equation

$$
\int_{\alpha}^{\beta} \log |t-x| d \lambda(t) \equiv Q(x)+\text { const }, \quad x \in[\alpha, \beta],
$$

for $\lambda, \lambda([\alpha, \beta])=1$. As was first noticed by A. A. Gonchar and E. A. Rakhmanov [20, it follows from (28) that at the end points of the support of the equilibrium measure, one has $\left(V^{\lambda}+Q\right)^{\prime}(x)=0$. In some cases, this observation allows one to find equations for the end points of the support. We remark that the problem of explicitly finding the end points of the unknown interval: 2 for a given polynomial $Q(x)$ is still unsolved in general (see [9]).

\footnotetext{
${ }^{2}$ The number of those intervals is also not known in advance.
} 


\section{Proof of Theorem 1}

3.1. Recall first some formulas and relations obtained in [44] in the case of a fixed (i.e., independent of $n$ ) weight

$$
\sigma(x)=\frac{i \rho(x)}{2 \pi W^{+}(x)}>0, \quad x \in E,
$$

where $\rho(x)$ is a real-valued function holomorphic on $E$ such that $\sigma(x)>0$ on $E$ (notice that $\rho(x)$ is positive for $x \in E_{g+1}$; on the remaining intervals it has the same sign as $\left.W^{+}(x) / i\right)$. Those formulas and relations are exact. Thus, to find their analogs in the case of a varying weight $\sigma_{n}$ of the form (11), we need to replace, in some formulas, $\rho$ by $\rho_{n}$; find a new explicit description of the normalized (see (35) Nuttall psi-function $\Psi_{n}(z ; n):=\Psi_{n}\left(z ; \rho_{n}\right)$; use this to determine its leading (i.e., of degree $\left.z^{n}\right)$ coefficient $\varkappa_{n}(n)$; and, using Theorem 2 (see the asymptotic formula (16) ), find the asymptotics of the leading coefficient of the orthonormal polynomial $q_{n}(z ; n)=\alpha_{n}(n) z^{n}+\ldots$ from the formula

$$
\alpha_{n}(n)=\varkappa_{n}(n)(1+o(1)), \quad n \rightarrow \infty .
$$

Let us recall the standard definition (see [31, 42, [44]) of the Nuttall psi-function $\Psi_{n}(\boldsymbol{z})=\Psi_{n}(\boldsymbol{z} ; \rho)$ defined on the two-sheeted hyperelliptic Riemann surface $\mathfrak{R}: W^{2}=$ $H(z), H(z)=\prod_{j=1}^{2 p}\left(z-e_{j}\right)$ of genus $g=p-1$. Fix an arbitrary $n \in \mathbb{N}, n \geq g$, and consider for the function $\rho(x)$ on the Riemann surface $\mathfrak{R}$, the following Riemann boundary value problem.

Problem 1. For a fixed $n \in \mathbb{N}, n \geq g$, find a function $\Psi_{n}(\boldsymbol{z})=\Psi_{n}(\boldsymbol{z} ; \rho)$ such that:

1) $\Psi_{n}$ is (piecewise) meromorphic on $\mathfrak{R} \backslash \mathcal{L}=D^{(1)} \sqcup D^{(2)}$;

2) divisor $\left(\Psi_{n}\right)=(n-g) \infty^{(2)}+\boldsymbol{z}_{1}+\ldots+\boldsymbol{z}_{g}-n \infty^{(1)}$;

3) the boundary condition $\rho(x) \Psi_{n}^{(1)}(\boldsymbol{x})=\Psi_{n}^{(2)}(\boldsymbol{x}), \boldsymbol{x} \in \mathcal{L}$, holds on $\mathcal{L}$.

In 2), the points $\boldsymbol{z}_{j}$ (the "free" zeroes of the psi-function) depend only on $n$ and on $\rho$. In 3$)$, by $\Psi_{n}^{(1)}(\boldsymbol{x})$ we understand the nontangent limit values of $\Psi_{n}(\boldsymbol{z})$ when $D^{(1)} \ni \boldsymbol{z} \rightarrow$ $\boldsymbol{x} \in \mathcal{L}$, and $\Psi_{n}^{(2)}(\boldsymbol{x})$ is interpreted in a similar way.

In our case, when the compact set $E$ consists of a finite number of intervals on the real line (recall that $\operatorname{proj} \mathcal{L}=E$ ), the function $\Psi_{n}$ solving Problem 1 always exists (see [42, [43, [44]). Moreover, since the genus $g$ of $\mathfrak{R}$ is positive, the zeroes and the poles of $\Psi_{n}$ on $\mathfrak{R}$ satisfy certain relations similar to Abel's relations for meromorphic functions on $\mathfrak{R}$ (when $\rho(x) \equiv 1$ these relations coincide with Abel's relations), and the divisor $d=\boldsymbol{z}_{1}+\ldots+\boldsymbol{z}_{g}$ is a solution of a special Jacobi inversion problem (14) (see also (40) below).

The defining properties of the Nuttall psi-function (Problem 10 show that

$$
\Psi_{n}\left(z^{(1)}\right) \Psi_{n}\left(z^{(2)}\right) \equiv \mathrm{const} \prod_{j=1}^{g}\left(z-z_{j}\right)
$$

for $z \in \mathbb{C} \backslash E$. Henceforth, we shall use the (uniquely defining the psi-function) normalization

$$
\Psi_{n}\left(z^{(1)}\right) \Psi_{n}\left(z^{(2)}\right) \equiv \prod_{j=1}^{g}\left(z-z_{j}\right)
$$

and the leading (i.e., of degree $z^{n}$ ) coefficient of $\Psi_{n}\left(z^{(1)}\right)$ is positive. We shall say that such a psi-function is normalized. We remark that the polynomial $X_{g}(z ; n):=$ $\prod_{j=1}^{g}\left(z-z_{j}\right)$ is in fact an unknown "polynomial parameter" of Problem 1 
Notice that the choice of the function $\rho$ such that the corresponding (see (33) ) function $\sigma(x)>0$ on $E$ yields the following result (see [44, Appendix B, part 5]).

Lemma 3.1. If a weight $\sigma(x)$ is positive on $E$ and defined by (33), then for any $n \geq g$ the points $z_{j}(n)=\operatorname{proj} \boldsymbol{z}_{j}(n)$ satisfying the Jacobi inversion problem (40) belong to the closed gaps between the intervals $E_{j}: z_{j}(n) \in\left[e_{2 j}, e_{2 j+1}\right], j=1,2, \ldots, g$. Thus, for any $n \geq g$ the divisor $d(n)=\boldsymbol{z}_{1}(n)+\ldots+\boldsymbol{z}_{g}(n)$ is non-special; i.e., the solution of the corresponding Jacobi inversion problem is unique.

This fact will play an important role in our arguments below.

Let $d \Omega_{k}, k=1, \ldots, g$, be an $a$-normalized basis of holomorphic abelian differentials (1-forms) on $\mathfrak{R}: \oint_{\boldsymbol{a}_{j}} d \Omega_{k}=\delta_{k j}, k, j=1, \ldots, g$. The abelian integrals $\Omega_{k}(\boldsymbol{z})=$ $\int_{e_{2 g+2}}^{z} d \Omega_{k}$ are defined on $\mathfrak{R}$ uniquely modulo their $a$ - and $b$-periods; henceforth we shall assume that when integrating abelian differentials the initial point is $e_{2 g+2}$. Since $\Omega_{k}^{\prime}(\boldsymbol{z})=s_{k}(z) / W(\boldsymbol{z})$, where $s_{k}$ is a polynomial of degree $g-1$, the notation $d \Omega_{k}^{ \pm}(x)=$ $s_{k}(x) d x / W^{ \pm}(x)$ makes sense for $x \in E$.

For any two distinct points $\boldsymbol{z}_{1}$ and $\boldsymbol{z}_{2}$ of the Riemann surface $\mathfrak{R}$, there exists an abelian differential of the third kind, holomorphic everywhere on $\mathfrak{R}$ except those two points, where it has simple poles with residues +1 and, respectively, -1 . Such a differential is said to be normal. A normal differential is determined uniquely whenever all of its $a$ periods vanish; we shall call such a differential an a-normalized abelian differential of the third kind and denote it by $d \Omega\left(\boldsymbol{z}_{1}, \boldsymbol{z}_{2} ; \boldsymbol{z}\right)$, where $\boldsymbol{z}$ is a variable on the Riemann surface $\Re$. Another method to uniquely define (i.e., normalize) a differential of the third kind is to require that all of its periods be pure imaginary. For such differentials we shall use the notation $d G\left(\boldsymbol{z}_{1}, \boldsymbol{z}_{2} ; \boldsymbol{z}\right)$, where we assume that the residues at $\boldsymbol{z}_{1}$ and $\boldsymbol{z}_{2}$ are equal to -1 and, respectively, +1 . An example of this sort is the differential of a complex Green's function with singularity at the infinite point $G(z, \infty)=g(z, \infty)+i g^{*}(z, \infty)$ of a non-simply connected domain $D=\overline{\mathbb{C}} \backslash E$ (here $g^{*}(z, \infty)$ is the harmonic conjugate of Green's function $g(z, \infty))$. The function $G(z, \infty)$ is multi-valued in $D$ and

$$
G(z, \infty)=\int_{e_{2 g+2}}^{z} \frac{P_{g}(\zeta)}{\sqrt{H(\zeta)}} d \zeta, \quad z \in D,
$$

where $P_{g}(z)=P_{g}(z ; H)=z^{g}+\ldots$ is a real polynomial all of whose roots lie in the gaps $\left(e_{2 j}, e_{2 j+1}\right), j=1, \ldots, g$. The differential $d G=d G\left(\infty^{(1)}, \infty^{(2)} ; \boldsymbol{z}\right)=P_{g}(z) d z / W(\boldsymbol{z})$ is defined on the entire $\mathfrak{R}$ and has pure imaginary periods and simple poles at $\boldsymbol{z}=$ $\infty^{(1)}$ and $\boldsymbol{z}=\infty^{(2)}$ with residues -1 and, respectively, +1 . Thus, the function $u(\boldsymbol{z})=$ $\operatorname{Re} G(\boldsymbol{z}, \infty)$, where $G(\boldsymbol{z}, \infty)=\int_{e_{2 g+2}}^{\boldsymbol{z}} d G$, is single-valued on $\mathfrak{R}$, and for $\boldsymbol{z}=z^{(1)}$ we have $u\left(z^{(1)}\right)=g(z, \infty)$. The function $u(\boldsymbol{z})$ defines a natural continuation of Green's function $g(z, \infty)$ to the entire Riemann surface $\mathfrak{R}: g(\boldsymbol{z}, \infty)=u(\boldsymbol{z})$.

By $\omega_{k}(z), k=1, \ldots, p$, we shall denote the harmonic measure (at $z \in D$ ) of the interval $E_{k}$ with respect to the domain $D$.

In terms of the Riemann surface $\mathfrak{R}$, the Nuttall psi-function has the following explicit representation (see, first of all, 42], as well as [43], 44]):

$$
\Psi_{n}(\boldsymbol{z}):=\Psi_{n}(\boldsymbol{z} ; \rho)=\Phi(\boldsymbol{z})^{n-g} e^{A(\boldsymbol{z} ; \rho)} \mathcal{F}_{n}(\boldsymbol{z}), \quad \boldsymbol{z} \in \mathfrak{R} \backslash \mathcal{L}
$$

We want to explain this representation. In formula (36),

$$
\Phi(\boldsymbol{z})=e^{G(\boldsymbol{z}, \infty)}
$$


is the so-called (multi-valued) function with a single-valued modulus, $\left|\Phi\left(z^{(1)}\right)\right|>1$, $\left|\Phi\left(z^{(2)}\right)\right|<1$. The function 3

$$
A(\boldsymbol{z} ; \rho)=W(\boldsymbol{z})\left\{\frac{1}{2 \pi i} \int_{E} \frac{\log \rho(x)}{z-x} \frac{d x}{W^{+}(x)}+\frac{1}{2 \pi i} \sum_{k=1}^{g} v_{k} \int_{E_{k}} \frac{1}{x-z} \frac{d x}{W^{+}(x)}\right\}+\frac{1}{2} c_{g+1}(n),
$$

where $z \notin E, v_{k}=2 \int_{E} \log \rho(x) d \Omega_{k}^{+}(x)$, the quantity $c_{g+1}=c_{g+1}(n)$ is defined by the relation $e^{c_{g+1}}=\prod_{j=1}^{g}\left(e_{2 g+2}-z_{j}\right)$, and the points $z_{j}=z_{j}(n)$ are the solutions of the Jacobi inversion problem (40). When the weight $\rho$ is fixed, the function $A$ depends on $n$ only by way of the quantity $c_{g+1}(n)$, which "cancels out" later on when the required asymptotic formula (see (44)) is derived, and for this reason we drop the dependence on $n$ in (38). We remark that the function $\exp \{A(\boldsymbol{z} ; \rho)\}$ is a multi-valued analog of the classical Szego" function $D(z ; \rho)$ for the case of a single interval $E=[-1,1]$,

$$
D(z ; \rho)=\exp \left\{\frac{\sqrt{z^{2}-1}}{2 \pi} \int_{-1}^{1} \frac{\log \rho(x)}{z-x} \frac{d x}{\sqrt{1-x^{2}}}\right\}, \quad z \notin[-1,1] .
$$

The function $\mathcal{F}_{n}(\boldsymbol{z})$ is also multi-valued on $D$ and has the following representation:

$$
\mathcal{F}_{n}(\boldsymbol{z})=\exp \left[\sum_{j=1}^{g} \Omega\left(\boldsymbol{z}_{j}, \infty^{(1)} ; \boldsymbol{z}\right)+2 \pi i \sum_{k=1}^{g} \theta_{k} \Omega_{k}(\boldsymbol{z})\right],
$$

where $\Omega\left(\boldsymbol{z}_{j}, \infty^{(1)} ; \boldsymbol{z}\right)$ is an integral of an $a$-normalized abelian differential of the third kind, the quantities $4 \theta_{k}=\theta_{k}(n)=\ell_{k}(n)+\left\{(n-g) \omega_{k}(\infty)\right\}$, where the integers $\ell_{k}(n)$ are uniformly bounded as $n \rightarrow \infty$, and the divisor $d=\boldsymbol{z}_{1}+\ldots+\boldsymbol{z}_{g}$, where $\boldsymbol{z}_{j}=\boldsymbol{z}_{j}(n)$, is the (unique) solution of the Jacobi inversion problem

(40) $\sum_{j=1}^{g} \Omega_{k}\left(\boldsymbol{z}_{j}\right) \equiv-\frac{i}{\pi} \int_{E} \log \rho(x) d \Omega_{k}^{+}(x)-\sum_{j=1}^{g}\left\{\left(n-g+\frac{1}{2}\right) \omega_{j}(\infty)\right\} B_{k j}, \quad k=1, \ldots, g$

(here the symbol $\equiv$ denotes congruence modulo $a$ - and $b$-periods). We remark that the uniformly bounded integers $\ell_{k}(n), k=1, \ldots, g$, appear in (39) because of the nonuniqueness of the abelian integrals $\Omega_{k}(\boldsymbol{z})$ for $\boldsymbol{z} \in \partial \widetilde{\mathfrak{R}}$ along the paths lying in $\widetilde{\mathfrak{R}}$, where $\widetilde{\mathfrak{R}}$ is the Riemann surface $\mathfrak{R}$ with a cut (for details, see [44, Appendices A and B]). Notice that for a given positivity condition on the weight $\sigma(x)$ of the form (33) for any $n \geq g$, the points $\boldsymbol{z}_{1}, \ldots, \boldsymbol{z}_{g}$ satisfying (40) are such that the corresponding points $z_{j}(n)=$ $\operatorname{proj} z_{j}(n)$ lie in the gaps between the intervals $E_{j}: z_{j}(n) \in\left[e_{2 j}, e_{2 j+1}\right], j=1,2, \ldots, g$ (see Lemma 3.1). Thus, for any $n \geq g$, the divisor $d(n)=\boldsymbol{z}_{1}(n)+\ldots+\boldsymbol{z}_{g}(n)$ is nonspecial, and therefore the solution of the corresponding Jacobi inversion problem (40) is always unique. This is true whether or not the weight $\sigma$ is varying.

Since the function $\rho$ is holomorphic and doesn't vanish on $E$, the right-hand side of (36) can be viewed as a holomorphic function even when $z \in D^{(1)} \sqcup \mathcal{L}$ for the divisor $d=\boldsymbol{z}_{1}+\ldots+\boldsymbol{z}_{g}$ satisfying (40). Thus, the function $\Psi_{n}(\boldsymbol{z}), \boldsymbol{z} \in D^{(1)} \sqcup \mathcal{L}$, should naturally be understood as the right-hand side of (36). The same is true for $\Psi_{n}(\boldsymbol{z})$ when $z \in D^{(2)} \sqcup \mathcal{L}$. On $\mathcal{L}$, these two holomorphic continuations do not agree: they satisfy the boundary condition 3 ), where, in general, $\rho \neq \equiv 1$.

A derivation of the explicit formulas (36)-(40) for the psi-function can be found in 44 , Appendix B].

\footnotetext{
${ }^{3}$ Here and elsewhere we choose the principal branch of the logarithm.

${ }^{4}$ Here and in (40), the symbol $\{\cdot\}$ denotes the fractional part of the corresponding number.
} 
3.2. In this subsection, for the sake of brevity, instead of $\Psi_{n}\left(\boldsymbol{z} ; \rho_{n}\right), \varkappa_{n}(n)$, and $z_{j}(n)$, we shall sometimes write $\Psi_{n}(\boldsymbol{z}), \varkappa_{n}$ and $z_{j}$. Let

$$
\Psi_{n}(z):=\Psi_{n}\left(z^{(1)} ; \rho_{n}\right)=\varkappa_{n}(n) z^{n}+O\left(z^{n-1}\right), \quad z \rightarrow \infty .
$$

Because of the normalization condition $\Psi_{n}\left(z^{(1)}\right) \Psi_{n}\left(z^{(2)}\right) \equiv \prod_{j=1}^{g}\left(z-z_{j}\right)$, it follows from (41) that

$$
\frac{\Psi_{n}\left(z^{(1)}\right)}{\Psi_{n}\left(z^{(2)}\right)}=\frac{\Psi_{n}\left(z^{(1)}\right)^{2}}{\Psi_{n}\left(z^{(2)}\right) \Psi_{n}\left(z^{(1)}\right)}=\varkappa_{n}^{2} z^{2 n-g}+O\left(z^{2 n-g-1}\right), \quad z \rightarrow \infty .
$$

Thus,

$$
\varkappa_{n}^{2}=\lim _{z \rightarrow \infty} \frac{\Psi_{n}\left(z^{(1)}\right)}{\Psi_{n}\left(z^{(2)}\right)} z^{g-2 n},
$$

and we may assume that $z>0$. We now proceed along the arguments of [44, formulas (53)-(59)]. Using the explicit formulas (36)-(40) we have

$$
\frac{\Psi_{n}\left(z^{(1)}\right)}{\Psi_{n}\left(z^{(2)}\right)}=\Phi^{2 n-2 g}(z) e^{A\left(z^{(1)} ; \rho\right)-A\left(z^{(2)} ; \rho\right)} \frac{\mathcal{F}_{n}\left(z^{(1)}\right)}{\mathcal{F}_{n}\left(z^{(2)}\right)} .
$$

In (44), the first factor behaves, when $z \rightarrow \infty$, as $(\operatorname{cap} E)^{2 g-2 n} z^{2 n-2 g}+O\left(z^{2 n-2 g-1}\right)$. In 44, only the case of a fixed weight $\rho$ was considered, and therefore the second factor did not depend on $n$ (the quantity $c_{g+1}(n) / 2$ from (38) cancels out when passing to (44)). Now we have to take into account that instead of $\rho$ we have $\rho_{n}$.

First, consider the third factor, i.e., the function

$$
\mathcal{F}_{n}\left(z^{(1)}\right) / \mathcal{F}_{n}\left(z^{(2)}\right)=\exp \left(\varphi_{n}(z)\right),
$$

where (see (39))

$$
\begin{aligned}
\varphi_{n}(z)=\sum_{j=1}^{g}\left[\Omega\left(\boldsymbol{z}_{j}(n), \infty^{(1)} ; z^{(1)}\right)-\Omega\left(\boldsymbol{z}_{j}(n)\right.\right. & \left.\left., \infty^{(1)} ; z^{(2)}\right)\right] \\
& +2 \pi i \sum_{j=1}^{g} \theta_{j}(n)\left(\Omega_{j}\left(z^{(1)}\right)-\Omega_{j}\left(z^{(2)}\right)\right) .
\end{aligned}
$$

The following formula for $\varphi_{n}(z)$ was obtained in [44, formulas (57)-(59)] using the Riemann conditions:

$$
\varphi_{n}(z)=-\sum_{j=1}^{g}\left[g\left(\boldsymbol{z}_{j}, z\right)-g\left(z, \infty^{(1)}\right)\right]+i \beta(n)+C_{1}(z)
$$

where $\beta(n) \in \mathbb{R}$, and the function $C_{1}(z)$ did not depend on $n$. In the case of the varying weight $\rho=\rho_{n}$ considered here, the function $C_{1}(z)$, as well as the function $A\left(z ; \rho_{n}\right)$, depends on $n$ by way of the varying weight. Thus, for the derivation of an asymptotic formula for the coefficient $\varkappa_{n}=\varkappa_{n}(n)$ along the lines of [44, we need to take into account the dependence of the functions $C_{1}(z)=C_{1}\left(z ; \rho_{n}\right)$ and $A\left(z ; \rho_{n}\right)$ on the variable $n$.

Consider first $C_{1}(z)$. It follows from [44, formulas (54)-(58)] that

$$
\begin{aligned}
C_{1}(z) & =-2 \pi i \sum_{k=1}^{g} \omega_{k}(z) \cdot \frac{1}{\pi i} \int_{E} \log \rho_{n}(x) d \Omega_{k}^{+}(x) \\
& =-2 \sum_{k=1}^{g} \omega_{k}(z) \int_{E} \log \rho_{n}(x) d \Omega_{k}^{+}(x) .
\end{aligned}
$$


Thus,

$$
\begin{aligned}
C_{1}(\infty) & =-2 \sum_{k=1}^{g} \omega_{k}(\infty) \int_{E} \log \rho_{n}(x) d \Omega_{k}^{+}(x) \\
& =-2 \int_{E} \log \rho_{n}(x)\left\{\sum_{k=1}^{g} \omega_{k}(\infty) d \Omega_{k}^{+}(x)\right\} .
\end{aligned}
$$

Since $i \beta(n)$ is pure imaginary, we have

$$
\left|\exp \left\{\varphi_{n}(z)\right\} / z^{g}\right|_{z=\infty}=\frac{1}{(\operatorname{cap} E)^{g}} \exp \left\{-\sum_{j=1}^{g} g\left(\boldsymbol{z}_{j} ; \infty\right)+C_{1}(\infty)\right\},
$$

where $C_{1}(\infty)$ is given by (48).

We now examine the second factor in (44) or, more precisely, the difference

$$
A\left(z^{(1)}\right)-\left.A\left(z^{(2)}\right)\right|_{z=\infty}=2\left(A\left(\infty ; \rho_{n}\right)-\frac{1}{2} c_{g+1}(n)\right):=2 \widetilde{A}(\infty)
$$

(see (38)). The function $A\left(z ; \rho_{n}\right)$, constructed in [44] (for a fixed $n$ ), is holomorphic in a neighborhood of $z=\infty$, and, asymptotically at infinity, $W(z) \sim z^{g+1}, z \rightarrow \infty$. Therefore, the formulas (38) imply that the first $g$ moments of the function enclosed in curly brackets in (38) are necessarily zero; i.e., the following "orthogonality conditions" hold:

$$
\int_{E} \log \rho_{n}(x) x^{s} \frac{d x}{W^{+}(x)}-\sum_{k=1}^{g} v_{k} \int_{E_{k}} x^{s} \frac{d x}{W^{+}(x)}=0, \quad s=0, \ldots, g-1 .
$$

Thus, (38) and (50) imply that

$$
2 \widetilde{A}(\infty)=\frac{1}{\pi i}\left\{\int_{E} \log \rho_{n}(x) x^{g} \frac{d x}{W^{+}(x)}-\sum_{k=1}^{g} v_{k} \int_{E_{k}} x^{g} \frac{d x}{W^{+}(x)}\right\} .
$$

Consider $d \Omega_{A}(\boldsymbol{z})=d \Omega\left(\infty^{(1)}, \infty^{(2)} ; \boldsymbol{z}\right)$, a standard $a$-normalized abelian differential of the third kind. It is of the form $d \Omega_{A}(\boldsymbol{z})=d \Omega\left(\infty^{(1)}, \infty^{(2)} ; \boldsymbol{z}\right)=r_{g}(z) / W(z)$, where $r_{g}(z)=-z^{g}+\ldots$ is a polynomial of degree $g$ with leading coefficient -1 . Therefore,

$$
\int_{E_{k}} d \Omega_{A}^{+}(x)=0, \quad k=1, \ldots, g,
$$

and in view of (50), condition (51) becomes

$$
2 \widetilde{A}(\infty)=-\frac{1}{\pi i} \int_{E} \log \rho_{n}(x) d \Omega_{A}^{+}(x) .
$$

Using the identity [44, formula (A.3)]

$$
d G(\boldsymbol{\zeta})+2 \pi i \sum_{k=1}^{g} \omega_{k}(\infty) d \Omega_{k}(\boldsymbol{\zeta})+d \Omega_{A}(\boldsymbol{\zeta}) \equiv 0
$$

we deduce from (48) and (52) that

$$
\begin{aligned}
C_{1}(\infty)+2 \widetilde{A}(\infty) & =-\frac{1}{\pi i} \int_{E} \log \rho_{n}(x)\left\{2 \pi i \sum_{k=1}^{g} \omega_{k}(\infty) d \Omega_{k}^{+}(x)+d \Omega_{A}^{+}(x)\right\} \\
& =\frac{1}{\pi i} \int_{E} \log \rho_{n}(x) d G^{+}(x) .
\end{aligned}
$$

Since

$$
-\frac{1}{\pi i} d G^{+}(x)=d \gamma(x), \quad x \in E,
$$


where a $d \gamma(x)=d \gamma_{E}(x)$ is a Robin measure (i.e., an equilibrium measure with respect to $Q \equiv 0$ ) for $E$, it follows from (54) that

$$
C_{1}(\infty)+2 \widetilde{A}(\infty)=-\int_{E} \log \rho_{n}(x) d \gamma(x)=\text { const }+2 n \int_{E} Q(x) d \gamma(x),
$$

where const $=\operatorname{const}\left(p_{g}\right)$ depends only on the auxiliary polynomial $p_{g}$. Using the equilibrium condition $Q(x)+V^{\lambda_{Q}}(x) \equiv w_{Q}$ on $E$ and changing the order of integration, we deduce from (55) that

$$
C_{1}(\infty)+2 \widetilde{A}(\infty)=\operatorname{const}\left(p_{g}\right)+2 n\left(w_{Q}-w_{0}\right)
$$

where $w_{0}$ is the Robin constant for the compact set $E$ (with respect to $Q \equiv 0$ ).

Since cap $E=e^{-w_{0}}$, the relations (43), (44), (46), (49), and (56) yield the following formula for $\varkappa_{n}(n)$ :

$$
\varkappa_{n}^{2}(n)=\operatorname{const}\left(p_{g}\right) \cdot(\operatorname{cap} E)^{g} \cdot e^{2 n w_{Q}} \cdot \exp \left\{-\sum_{j=1}^{g} g\left(\boldsymbol{z}_{j}(n), \infty\right)\right\},
$$

where the divisor $d(n)=\boldsymbol{z}_{1}(n)+\ldots+\boldsymbol{z}_{g}(n)$ is a solution of the Jacobi inversion problem (40) with $\rho$ changed to $\rho_{n}$. Thus, in accordance with Lemma 3.1, all $z_{j}=\operatorname{proj} \boldsymbol{z}_{j}(n)$ lie in the gaps between the intervals that make up E. Directly from (57) and Theorem 2, we have the asymptotic formula (13) for the leading coefficient $\alpha_{n}(n)$ of the orthonormal polynomial $q_{n}(z ; n)=\alpha_{n}(n) z^{n}+\ldots$ as $n \rightarrow \infty$.

To finish the proof of Theorem 1, we need to rewrite (40) in the form (14). This will be done in 3.3 .

3.3. Thus, our goal is to rewrite, in the case $\rho=\rho_{n}, \rho_{n}(x):=p_{g}(x) e^{-2 n Q(x)}$ (see (11)), the relations (cf. (40))

$$
\sum_{j=1}^{g} \Omega_{k}\left(\boldsymbol{z}_{j}\right) \equiv-\frac{i}{\pi} \int_{E} \log \rho_{n}(x) d \Omega_{k}^{+}(x)-\sum_{j=1}^{g}\left\{n \omega_{j}(\infty)\right\} B_{k j}+c_{k}, \quad k=1, \ldots, g,
$$

where the constants $c_{k}$ are independent of $n$, in the form (14). Recall that, in accordance with our convention, the abelian integral $\Omega_{k}(\boldsymbol{z})$ is defined as follows:

$$
\Omega_{k}(\boldsymbol{z})=\int_{e_{2 g+2}}^{\boldsymbol{z}} d \Omega_{k}(\boldsymbol{\zeta}), \quad k=1, \ldots, g .
$$

Thus, if for $\boldsymbol{z}=z^{(1)}$ we integrate along a path contained in the domain $D^{(1)}$, the periods of $\Omega_{k}\left(z^{(1)}\right)$ would be pure imaginary. Therefore, $\operatorname{Im} \Omega_{k}\left(z^{(1)}\right)$ is a single-valued function on $D^{(1)}$ for each $k=1, \ldots, g$.

Let $\widetilde{M}_{1}(E)$ be the totality of all measures $\mu \in M_{1}(E)$ such that the integrals $\int_{E} V^{\mu}(x) d \Omega_{k}^{+}(x)$ exist for all $k=1, \ldots, g$. We need the following.

Lemma 3.2. For any unit measure $\mu \in \widetilde{M}_{1}(E)$ with support in $E$,

$$
\int_{E} V^{\mu}(x) d \Omega_{k}^{+}(x)=-\pi \int_{E} \operatorname{Im} \Omega_{k}^{+}(t) d \mu(t)+\text { const }_{k},
$$

where const $_{k}, k=1, \ldots, g$, are constants independent of the measure $\mu$.

Remark 3.1. The relation (60) should be interpreted in the standard way: the left-hand side exists if and only if the right-hand side does, and the two coincide.

Proof of Lemma 3.2. When $z \notin E$, let $\gamma$ denote the family of $p$ non-intersecting ovals containing the intervals $E_{1}, \ldots, E_{p}$ inside themselves and such that $z$ lies outside those ovals (the ovals are traversed in such a way that their interiors remain on the right), and 
let $f_{k}(z):=\int_{\gamma} \log (z-t) d \Omega_{k}(t)$. Then, since the differentials $d \Omega_{k}(z)$ are real on $E$, for $z \notin E$ we have

$$
\int_{E} \log |z-x| d \Omega_{k}^{+}(x)=\frac{1}{2} \operatorname{Re} \int_{\gamma} \log (z-t) d \Omega_{k}(t)=\frac{1}{2} \operatorname{Re} f_{k}(z) .
$$

For the multi-valued function $f_{k}(z)$ we have

$$
f_{k}^{\prime}(z)=\int_{\gamma} \frac{1}{z-t} d \Omega_{k}(t)=-2 \pi i \Omega_{k}^{\prime}(z), \quad z \notin E,
$$

where $\Omega_{k}^{\prime}(z)=s_{k}(z) / W(z)$ and $s_{k}$ is a polynomial of degree $g-1$. Therefore, it follows from (62) that

$$
f_{k}(z)=-2 \pi i \Omega_{k}(z)+\text { const }_{k} \quad(\bmod \text { periods }) .
$$

Now, for any measure $\mu \in \widetilde{M}_{1}(E)$, it follows from the Fubini theorem (assuming that the corresponding integrals exist) that

$$
\begin{aligned}
\int_{E} V^{\mu}(x) d \Omega_{k}^{+}(x) & =-\int_{E}\left(\int_{E} \log |x-t| d \Omega_{k}^{+}(x)\right) d \mu(t) \\
& =-\int_{E}\left(\lim _{s \rightarrow t^{+}} \int_{E} \log |x-s| d \Omega_{k}^{+}(x)\right) d \mu(t) \\
& =-\frac{1}{2} \int_{E} \operatorname{Re}\left\{f_{k}(t)\right\}^{+} d \mu(t)=-\frac{1}{2} \operatorname{Re} \int_{E} f_{k}^{+}(t) d \mu(t) \\
& =\pi \operatorname{Re}\left\{i \int_{E} \Omega_{k}^{+}(t) d \mu(t)+c_{k}\right\} \\
& =-\pi \int_{E} \operatorname{Im} \Omega_{k}^{+}(t) d \mu(t)+\widetilde{c}_{k}
\end{aligned}
$$

Lemma 3.2 is proved.

For any measures $\mu_{1}, \mu_{2} \in \widetilde{M}_{1}(E)$, it follows from (60) that

$$
\int_{E} V^{\mu_{1}-\mu_{2}}(x) d \Omega_{k}^{+}(x)=-\pi \int_{E} \operatorname{Im} \Omega_{k}^{+}(t) d\left(\mu_{1}-\mu_{2}\right)(t) .
$$

Since $\Omega_{k}^{+}(x)=-B_{k j}$ on the intervals $E_{j}, j=1, \ldots, g$, and $\Omega_{k}^{+}(x)$ is real on $E_{g+1}$, it follows from (65) that

$$
\int_{E} V^{\mu_{1}-\mu_{2}}(x) d \Omega_{k}^{+}(x)=\frac{\pi}{2} \sum_{j=1}^{g} \operatorname{Im} B_{k j}\left(\mu_{1}-\mu_{2}\right)\left(E_{j}\right) .
$$

Let us apply (66) to the equilibrium measures $\mu_{1}=\lambda$ and $\mu_{2}=\gamma$ (clearly, $\lambda, \gamma \in$ $\left.\widetilde{M}_{1}(E)\right)$. Taking into account that $V^{\lambda}(x)-V^{\gamma}(x) \equiv\left(w_{Q}-w_{0}\right)-Q(x), x \in E$, and $\int_{E} d \Omega_{k}^{+}(x)=0$ we have

$$
\begin{aligned}
\int_{E} Q(x) d \Omega_{k}^{+}(x) & =-\int_{E} V^{\lambda-\gamma}(x) d \Omega_{k}^{+}(x)=\frac{\pi}{2} \sum_{j=1}^{g} \operatorname{Im} B_{k j}\left(\gamma\left(E_{j}\right)-\lambda\left(E_{j}\right)\right) \\
& =\frac{\pi}{2} \sum_{j=1}^{g} \operatorname{Im} B_{k j}\left(\omega_{j}(\infty)-\lambda\left(E_{j}\right)\right) .
\end{aligned}
$$


Finally, it follows from (67) (and from the fact that the $B_{k j}$ are imaginary) that (see (58)), for the weight $\rho_{n}$ given by (15),

$$
\begin{aligned}
-\frac{i}{\pi} \int_{E} \log \rho_{n}(x) d \Omega_{k}^{+}(x) & =\frac{2 n i}{\pi} \int_{E} Q(x) d \Omega_{k}^{+}(x)+c_{k}\left(p_{g}\right) \\
& =n i \sum_{j=1}^{g} \operatorname{Im} B_{k j}\left(\gamma\left(E_{j}\right)-\lambda\left(E_{j}\right)\right)+c_{k}\left(p_{g}\right) \\
& =n \sum_{j=1}^{g} B_{k j}\left(\gamma\left(E_{j}\right)-\lambda\left(E_{j}\right)\right)+c_{k}\left(p_{g}\right) \\
& =n \sum_{j=1}^{g} B_{k j}\left(\omega_{j}(\infty)-\lambda\left(E_{j}\right)\right)+c_{k}\left(p_{g}\right) .
\end{aligned}
$$

Using (68) we rewrite (58) as follows (as before, the symbol $\equiv$ denotes congruence modulo $a$ - and $b$-periods):

$$
\begin{aligned}
\sum_{j=1}^{g} \Omega_{k}\left(\boldsymbol{z}_{j}\right) & \equiv-\frac{i}{\pi} \int_{E} \log \rho_{n}(x) d \Omega_{k}^{+}(x)-\sum_{j=1}^{g}\left\{n \omega_{j}(\infty)\right\} B_{k j}+c_{k} \\
& \equiv n \sum_{j=1}^{g} B_{k j}\left(\omega_{j}(\infty)-\lambda\left(E_{j}\right)\right)-n \sum_{j=1}^{g} \omega_{j}(\infty) B_{k j}+c_{k}+c_{k}\left(p_{g}\right) \\
& \equiv-n \sum_{j=1}^{g} B_{k j} \lambda\left(E_{j}\right)+\tilde{c}_{k}\left(p_{g}\right) \\
& \equiv-\sum_{j=1}^{g}\left\{n \lambda\left(E_{j}\right)\right\} B_{k j}+\tilde{c}_{k}\left(p_{g}\right), \quad k=1, \ldots, g .
\end{aligned}
$$

This proves (14) and thus Theorem 1.

\section{Some CONSEQUENCES AND REMARKS}

4.1. We begin with a remark.

Remark 4.1. The above results remain true if the weight $\rho_{n}(x)=e^{-2 n Q(x)}$ is replaced by the more general weight $\rho_{N}(x)=e^{-2 N Q(x)}$, where $N=n \pm 1, \pm 2, \ldots, \pm \ell$ and $\ell \in \mathbb{N}$ is fixed. Accordingly, the definition of the Nuttall psi-function has to be modified.

Problem 2. For a fixed $n \in \mathbb{N}, n \geq g$, and $N=n+\ell, \ell \in \mathbb{Z}$, find a function $\Psi_{n, N}(\boldsymbol{z}):=$ $\Psi_{n}\left(\boldsymbol{z} ; \rho_{N}\right)$ such that:

1) $\Psi_{n, N}$ is (piecewise) meromorphic on $\mathfrak{R} \backslash \mathcal{L}=D^{(1)} \sqcup D^{(2)}$;

2) the divisor $\left(\Psi_{n, N}\right)=(n-g) \infty^{(2)}+\boldsymbol{z}_{1}+\ldots+\boldsymbol{z}_{g}-n \infty^{(1)}$;

3) the boundary condition $\rho_{N}(x) \Psi_{n, N}^{(1)}(\boldsymbol{x})=\Psi_{n, N}^{(2)}(\boldsymbol{x}), \boldsymbol{x} \in \mathcal{L}$ holds on $\mathcal{L}$.

In 2), the points $\boldsymbol{z}_{j}=\boldsymbol{z}_{j}(n ; N)$ (the "free" zeroes of the psi-function) depend on $n$ and $N$, and in 3), by $\Psi_{n, N}^{(1)}(\boldsymbol{x})$ we understand the limit values of $\Psi_{n, N}(\boldsymbol{z})$ as $D^{(1)} \ni \boldsymbol{z} \rightarrow \boldsymbol{x} \in \mathcal{L}$, with a similar interpretation for $\Psi_{n, N}^{(2)}(\boldsymbol{x})$. In this case, Theorem 2 (more precisely, the strong asymptotic formula (16)) rewrites as

$$
q_{n}(z ; N)=\Psi_{n, N}(z)(1+o(1)), \quad n \rightarrow \infty .
$$

As follows directly from Remark 4.1 and formulas (13)-(14), we have the following qualitative description of the leading coefficient $\alpha_{n}(n)$.

Corollary 4.1. The parameters $a_{n}(n)=\alpha_{n-1}(n) / \alpha_{n}(n)$ and $b_{n}(n)$ from the standard trinomial, with respect to the first index, recurrence relation

$$
Q_{n}(x ; n)=\left(x-b_{n}(n)\right) Q_{n-1}(x ; n)-a_{n-1}^{2}(n) Q_{n-2}(x ; n)
$$


for the monic polynomials $Q_{n-2}(x ; n), Q_{n-1}(x ; n), Q_{n}(x ; n)$ satisfy the following asymptotic relations ("trace formulas"; cf. [44, formulas (28)-(29)], [13, formula (1.65)], and [33. formula (41)]):

$$
\begin{gathered}
a_{n}(n)=\operatorname{cap} E \cdot \exp \left\{\frac{1}{2} \sum_{j=1}^{g} g\left(\boldsymbol{z}_{j}(n ; n), \infty\right)-\frac{1}{2} \sum_{j=1}^{g} g\left(\boldsymbol{z}_{j}(n-1 ; n), \infty\right)\right\}(1+o(1)), \\
b_{n}(n)=\frac{1}{2} \sum_{j=1}^{2 p} e_{j}-\sum_{j=1}^{p-1} z_{j}(n-1 ; n)+o(1), \quad n \rightarrow \infty
\end{gathered}
$$

where the divisors $d(n ; n)$ and $d(n-1 ; n)$ are related by the formula

$$
\left\{\sum_{j=1}^{g} \int_{\boldsymbol{z}_{j}(n-1 ; n)}^{\boldsymbol{z}_{j}(n ; n)}\right\} d \Omega_{k} \equiv-\sum_{j=1}^{g} \omega_{j}(\infty) B_{k j}, \quad k=1, \ldots, g .
$$

We remark that the formulas (71)-(73) do not carry any explicit information about the external field $Q$ and that they are identical to the formulas for weights independent of $n$. The formula (73) means that the relative displacement of the "adjacent" divisors $d(n ; n)$ and $d(n-1 ; n)$ is determined only by the geometric data, i.e., the harmonic measures $\omega_{j}(\infty)$ of the intervals $E_{j}$ that make up the compact set $E$.

Next, we mention a corollary of Theorem 2 (see (77)), which is naturally interpreted as an analog of a known corollary to a theorem of Rakhmanov on the asymptotic ratio for orthogonal polynomials (see [36, 38]), for the case of a varying weight. More precisely, one of the corollaries to Rakhmanov's theorem asserts that if $\mu^{\prime}=d \mu / d x>0$ almost everywhere on the interval $\Delta=[-1,1]$, then for the polynomials $q_{n}(x)=q_{n}(x ; \mu)$ orthonormal with respect to a (positive Borel) measure $\mu$,

$$
\int_{\delta} q_{n}^{2}(x) d \mu(x)=1, \quad n=1,2, \ldots
$$

one has

$$
q_{n}^{2}(x) d \mu(x) \stackrel{*}{\rightarrow} \frac{1}{\pi} \frac{d x}{\sqrt{1-x^{2}}}, \quad n \rightarrow \infty
$$

(the convergence in (74) is understood in the sense of weak convergence in the space of measures). Let $r_{n}(z)$ be the function of the second kind corresponding to the polynomial $q_{n}(x)$ (or, in a different terminology, the remainder function)

$$
r_{n}(z)=\int_{\Delta} \frac{q_{n}(x) d \mu(x)}{z-x}, \quad z \notin \Delta .
$$

Then it follows from (74) and (75) that, locally uniformly for $z \notin \Delta$,

$$
q_{n}(z) r_{n}(z)=\int_{\Delta} \frac{q_{n}^{2}(x) d \mu(x)}{z-x} \rightarrow \frac{1}{\pi} \int_{\Delta} \frac{1}{z-x} \frac{d \mu(x)}{\sqrt{1-x^{2}}}=\frac{1}{\sqrt{z^{2}-1}}, \quad n \rightarrow \infty .
$$

Now let $E=\bigsqcup_{j=1}^{p} E_{j}$ be the union of $p \geq 2$ non-intersecting intervals. Then, for some (rather general but more restrictive than in Rakhmanov's theorem) condition on the orthogonality measure for the corresponding orthonormal polynomials and the remainder functions, we have an asymptotic relation

$$
q_{n}(z) r_{n}(z)=\frac{\prod_{j=1}^{p-1}\left(z-z_{j}\right)}{\sqrt{H_{2 p}(z)}}+o(1), \quad n \rightarrow \infty .
$$

In (77), the points $z_{j}$ depend on $n$ and are obviously related to the solution of the Jacobi inversion problem of the form (40) for the corresponding non-varying weight, and $H_{2 p}(z)$ is a monic polynomial of degree $2 p$ with simple roots at the end points of the intervals $E_{j}$. 
The relation (77) is a direct consequence of the fact that, in the case of several intervals, in addition to the asymptotic formula for the orthonormal polynomials

$$
q_{n}(z)=\Psi_{n}\left(z^{(1)}\right)(1+o(1)), \quad n \rightarrow \infty,
$$

(see [44]) we have a similar formula for the remainder functions:

$$
r_{n}(z)=\frac{\Psi_{n}\left(z^{(2)}\right)}{\sqrt{H_{2 p}(z)}}(1+o(1)), \quad n \rightarrow \infty .
$$

Now (77) follows from (78) and (79), together with the identity (see (35))

$$
\Psi_{n}\left(z^{(1)}\right) \Psi_{n}\left(z^{(2)}\right)=\prod_{j=1}^{p-1}\left(z-z_{j}\right) .
$$

The formulas (77)-(79) can naturally be extended to the case of a varying weight, namely,

$$
\begin{gathered}
q_{n}(z ; n)=\Psi_{n}\left(z^{(1)} ; n\right)(1+o(1)), \quad r_{n}(z ; n)=\frac{\Psi_{n}\left(z^{(2)} ; n\right)}{\sqrt{H_{2 p}(z)}}(1+o(1)), \quad n \rightarrow \infty, \\
q_{n}(z ; n) r_{n}(z ; n)=\frac{\prod_{j=1}^{p-1}\left(z-z_{j}(n)\right)}{\sqrt{H_{2 p}(z)}}+o(1), \quad n \rightarrow \infty .
\end{gathered}
$$

In (81) the points $z_{j}(n)$ are obviously related to the solution of the Jacobi inversion problem of the form (14) for the corresponding varying weight.

4.2. We continue with more remarks.

Remark 4.2. The formula (70) is completely analogous to the well-known formula of Widom for a weight independent of $n$ (see [48, $\S 6$, Theorem $6.2 ; \S 9$, Theorem $9.1 ; \S 8$, Theorem 8.4], as well as [44, [28, and formula (86)). The conditions (87) on the divisor $d(n)=\boldsymbol{z}_{1}(n)+\ldots+\boldsymbol{z}_{g}(n)$ are equivalent to the following system of congruences (the Widom equations; see [48, $\S 8$, Theorem 8.4], as well as [37, where this formula is generalized to the case when a finite number of discrete masses are added to the weight):

$$
\sum_{j=1}^{g} \varepsilon_{j} \omega_{k}\left(z_{j}\right)=-2 n \lambda\left(E_{k}\right)+c_{k} \quad(\bmod 2),
$$

where $\omega_{k}(z)$ is the harmonic measure (at the point $z \in D$ ) of $E_{k}$ with respect to $\partial D \backslash E_{k}$, and $\varepsilon_{j}= \pm 1$. Notice that the system (82) uniquely determines the parameters $\varepsilon_{j}= \pm 1$ and the points $z_{j}$; thus the points $\boldsymbol{z}_{j}=\left(z_{j}, \pm\right)$ on the Riemann surface $\mathfrak{R}$ and the divisor $d(n)$ are also uniquely determined.

We remark that Widom's formula (86) is obtained from (70) by (formally) passing to the limit $Q(x) \equiv 0$ and by the corresponding substitutions $w_{Q} \mapsto w_{0}, \lambda\left(E_{j}\right) \mapsto \gamma\left(E_{j}\right)=$ $\omega_{j}(\infty)$, etc. Therefore, in some sense, the Widom formulas remain true in the case of a varying weight. Thus, they are of universal nature.

Remark 4.3. Let, as before, $\omega_{k}(z)$ be the harmonic measure of $E_{k}$. It is well-known that $\gamma\left(E_{j}\right)=\omega_{j}(\infty)$, cap $E=e^{-w_{0}}$ and that one has the following relation between the complex harmonic measures $\omega_{k}(\lambda)+i \omega_{k}^{*}(\lambda)$ and the abelian integrals (59) (see [48], [44]):

$$
\Omega_{j}(z)=-\frac{1}{2} \sum_{k=1}^{g} B_{k j}\left(\omega_{k}(z)+i \omega_{k}^{*}(z)\right), \quad z \in D,
$$

where $\omega_{k}^{*}(z)$ is the harmonic conjugate of $\omega_{k}(z)$. Thus, the formulas (13)-(14) rewrite as

$$
\alpha_{n}(n)=\text { const } \cdot e^{n w_{Q}} \cdot \exp \left\{-\frac{1}{2} \sum_{j=1}^{g} \varepsilon_{j} g\left(z_{j}, \infty\right)\right\}(1+o(1)), \quad n \rightarrow \infty,
$$




$$
\sum_{j=1}^{g} \varepsilon_{j} \omega_{k}\left(z_{j}\right)=-2 n \lambda\left(E_{j}\right)+c_{k} \quad(\bmod 2), \quad k=1, \ldots, g
$$

where $\varepsilon_{j}= \pm 1$. We remark that the system (85) uniquely determines the parameters $\varepsilon_{j}= \pm 1$ and the points $z_{j}$. Hence the points $\boldsymbol{z}_{j}=\left(z_{j}, \pm\right) \in \Re$ are also uniquely determined. Notice that the classical strong asymptotic formulas of Widom,

$$
\begin{gathered}
\alpha_{n}=e^{n w_{0}} \cdot \exp \left\{-\frac{1}{2} \sum_{j=1}^{g} \varepsilon_{j} g\left(z_{j}, \infty\right)\right\}(1+o(1)), \quad n \rightarrow \infty, \\
\sum_{j=1}^{g} \varepsilon_{j} \omega_{k}\left(z_{j}\right)=-2 n \omega_{j}(\infty)+c_{k} \quad(\bmod 2), \quad k=1, \ldots, g,
\end{gathered}
$$

are obtained from (84)-85) by (formally) passing to the limit $Q(x) \equiv 0$ and by the corresponding substitutions $w_{Q} \mapsto w_{0}, \lambda\left(E_{j}\right) \mapsto \gamma\left(E_{j}\right)=\omega_{j}(\infty)$, etc.

Remark 4.4. If we replace $n$ in the system (14) by a continuous parameter $t \in \mathbb{R}_{+}$ ("time"), then we can show that the motion of the divisor $d(t)=\boldsymbol{z}_{1}(t)+\ldots+\boldsymbol{z}_{g}(t)$ on the torus $\mathbb{T}^{g}$ corresponding to the system (14) (with $t$ in place of $n$ ) is governed by the following (generalized) system of Dubrovin differential equations (cf. [15] and [44, formula (26)]; as usual, the dot denotes the derivative with respect to $t$ ):

$$
\dot{\boldsymbol{z}}_{k}=-\frac{W\left(\boldsymbol{z}_{k}\right)}{\prod_{j \neq k}\left(z_{k}-z_{j}\right)}\left\{\int_{e_{2 g+2}}^{+\infty} \frac{\prod_{j \neq k}\left(x-z_{j}\right)}{W(x)} d x-\frac{2 i}{\pi} \int_{E} Q(x) \frac{\prod_{j \neq k}\left(x-z_{j}\right)}{W^{+}(x)} d x\right\},
$$

$k=1, \ldots, g$. Notice that the expression in curly brackets in (88) does not contain $\boldsymbol{z}_{k}$. The solution $d(n)$, needed for the asymptotic formula (70), can be obtained from the general solution $d(t)$ of (88) by substituting discrete values $t=n, n \in \mathbb{N}$. The parameters $2 \nu_{j}=2 \lambda\left(E_{j}\right), j=1, \ldots, g$, play the role of frequencies when the divisor $d(t)$ moves on the torus $\mathbb{T}^{g}$. If the frequencies $\nu_{1}, \ldots, \nu_{g}$ are rationally independent, then the winding line $\left\{d(t), t \in \mathbb{R}_{+}\right\}$defined by the divisor $d(t)$ is everywhere dense in the torus; if the numbers $\nu_{1}, \ldots, \nu_{g}, \nu_{g+1}$ are rationally independent (notice that, by definition, $\nu_{1}+\ldots+\nu_{g+1}=1$ ), then the integral "lattice" $\{d(n), n \in \mathbb{N}\}$ is everywhere dense in the winding line, and therefore in the entire torus $\mathbb{T}^{g}$.

We remark that in the case of a non-varying weight $\rho$ (i.e., $Q \equiv 0$ ) the formula (88) rewrites as (see [43])

$$
\dot{\boldsymbol{z}}_{k}=-\frac{W\left(\boldsymbol{z}_{k}\right)}{\prod_{j \neq k}\left(z_{k}-z_{j}\right)} \cdot\left\{\int_{e_{2 g+2}}^{+\infty} \frac{\prod_{j \neq k}\left(x-z_{j}\right)}{W(x)} d x\right\}, \quad k=1, \ldots, g
$$

(the expression in curly brackets in (89) does not contain $\boldsymbol{z}_{k}$ ). As before, the solution $d(n)$ needed for the Widom asymptotic formula can be obtained from the general solution $d(t)$ of (89) by substituting discrete values $t=n, n \in \mathbb{N}$. As in (88), the parameters $\nu_{j}=$ $\tau\left(E_{j}\right)=\omega_{j}(\infty), j=1, \ldots, g$, play the role of frequencies when the divisor $d(t)$ moves on the torus $\mathbb{T}^{g}$. If the frequencies $\nu_{1}, \ldots, \nu_{g}$ are rationally independent, then the winding line $\left\{d(t), t \in \mathbb{R}_{+}\right\}$defined by the divisor $d(t)$ is everywhere dense in the torus; if the numbers $\nu_{1}, \ldots, \nu_{g}, \nu_{g+1}$ are rationally independent (notice that, by definition, $\nu_{1}+\ldots+$ $\left.\nu_{g+1}=1\right)$, then the integral "lattice" $\{d(n), n \in \mathbb{N}\}$ is everywhere dense in the winding line, and therefore in the entire torus $\mathbb{T}^{g}$.

We remark that equations of type (89) appear not only in the theory of finite-gap integration but also in the study of other types of equations; see, for example, [14, Theorem 2.2, formulas (2.3)-(2.5)].

All that yields the following corollary to Theorem 1 
Corollary 4.2. If the quantities $\lambda\left(E_{1}\right), \ldots, \lambda\left(E_{g+1}\right)$ are rationally independent, then, as $n \rightarrow \infty$, the quantity

$$
2 \log \left(\alpha_{n}(n) \cdot e^{-n w_{Q}} \cdot \text { const }\right)
$$

oscillates near zero as $-\sum_{j=1}^{g} g\left(\boldsymbol{z}_{j}(n), \infty\right)$, and the set of limit points of the sequence (90) is the entire interval $[-A, A]$, where $A=\sum_{j=1}^{g} g\left(z_{j}^{*}, \infty\right)$.

Here, $z_{j}^{*} \in\left[e_{2 j}, e_{2 j+1}\right], j=1, \ldots, g$, are the critical points of Green's function $g(z, \infty)$ (see [18, [48]): $g^{\prime}\left(z_{j}^{*}, \infty\right)=0$.

Indeed, the independence of the "frequencies" $\lambda\left(E_{1}\right), \ldots, \lambda\left(E_{g+1}\right)$ implies that the points $\boldsymbol{z}_{1}(n), \ldots, \boldsymbol{z}_{g}(n)$ satisfying (14) are everywhere dense in the torus $\mathbb{T}^{g}$. Hence, in the relation (see (13) $)$

$$
\alpha_{n}^{2}(n) \cdot e^{-2 n w_{Q}}=\mathrm{const} \prod_{j=1}^{g} \exp \left\{-g\left(\boldsymbol{z}_{j}(n), \infty\right)\right\}(1+o(1)), \quad n \rightarrow \infty,
$$

they can be viewed as varying independently of each other. The corollary now follows.

Remark 4.5. Taking into account the geometric meaning of the harmonic measure (see [18, as well as [44, part 7, Appendix B]), the Widom equations (82) (after replacing $n$ by the time $t$ ) admit a natural interpretation: the divisor $d(t)=\boldsymbol{z}_{1}(t)+\ldots+\boldsymbol{z}_{g}(t)$ (necessarily satisfying (88) ) moves on the torus $\mathbb{T}^{g}$ with a constant "angular" velocity equal to $\left(2 \lambda\left(E_{1}\right), \ldots, 2 \lambda\left(E_{g}\right)\right)$ (for details, see [44, part 6, Appendix B]).

\section{REFERENCES}

[1] N. I. Ahiezer, Orthogonal polynomials on several intervals, Dokl. Akad. Nauk SSSR 134 (1960), no. 1, 9-12; English transl., Soviet Math. Dokl. 1 (1960), 989-992. MR0110916 (22:1784)

[2] N. I. Ahiezer, Continuous analogues of orthogonal polynomials on a system of intervals, Dokl. Akad. Nauk SSSR 141 (1961), no. 2, 263-266. MR0140970 (25:4383)

[3] A. I. Aptekarev, Asymptotic properties of polynomials orthogonal on a system of contours, and periodic motions of Toda chains, Mat. Sb. 125(167) (1984), no. 2(10), 231-258. MR764479 (86g:35166)

[4] A. I. Aptekarev, V. I. Buslaev, A. Martinez-Finkelshtein, and S. P. Suetin, Padé approximants, continued fractions, and orthogonal polynomials, Uspekhi Mat. Nauk 66 (2011), no. 6(402), 37-122; English transl., Russian Math. Surveys 66 (2011), no. 6, 1049-1131. MR2963451

[5] A. I. Aptekarev and A. B. J. Kuijlaars, Hermite-Padé approximations and ensembles of multiple orthogonal polynomials, Uspekhi Mat. Nauk 66 (2011), no. 6(402), 123-190; English transl., Russian Math. Surveys 66 (2011), no. 6, 1133-1199. MR2963452

[6] A. I. Aptekarev, V. G. Lysov, and D. N. Tulyakov, Random matrices with an external source and the asymptotics of multiple orthogonal polynomials, Mat. Sb. 202 (2011), no. 2, 3-56; English transl., Sb. Math. 202 (2011), no. 1-2, 155-206. MR2798785 (2012b:60019)

[7] A. I. Aptekarev and D. N. Tulyakov, Asymptotic regimes in the saturation zone for C-D-kernels for an ensemble of Meixner orthogonal polynomials, Uspekhi Mat. Nauk 66 (2011), no. 1(397), 181-182; English transl., Russian Math. Surveys 66 (2011), no. 1, 173-175. MR2841690(2012k:33015)

[8] A. I. Aptekarev and W. Van Assche, Scalar and matrix Riemann-Hilbert approach to the strong asymptotics of Padé approximants and complex orthogonal polynomials with varying weight, J. Approx. Theory 129 (2004), no. 2, 129-166. MR2078646 (2005m:41033)

[9] P. M. Bleher and B. Eynard, Double scaling limit in random matrix models and a nonlinear hierarchy of differential equations. Random matrix theory, J. Phys. A. 36 (2003), no. 12, 3085-3105. MR.1986409 (2005c:82041)

[10] V. S. Buyarov and E. A. Rakhmanov, On families of measures that are balanced in the external field on the real axis, Mat. Sb. 190 (1999), no. 6, 11-22; English transl., Sb. Math. 190 (1999), no. 5-6, 791-802. MR1719585 (2001b:31002)

[11] E. M. Chirka, Riemann surfaces, Lecture notes, MIAN Scientific Education Center, 1, 3-105, Moscow, 2006.

[12] P. Deift, Orthogonal polynomials and random matrices: a Riemann-Hilbert approach, Courant Lecture Notes in Mathematics, vol. 3, Amer. Math. Soc., Providence, RI, 1999. MR1677884 (2000g:47048) 
[13] P. Deift, T. Kriecherbauer, K. T.-R. McLaughlin, S. Venakides, and X. Zhou, Uniform asymptotics for polynomials orthogonal with respect to varying exponential weights and applications to universality questions in random matrix theory, Comm. Pure Appl. Math. 52 (1999), no. 11, 1335-1425. MR.1702716 (2001g:42050)

[14] V. Dragovich and M. Radnovic, Integrable billiards and quadrics, Uspekhi Mat. Nauk 65 (2010), no. 2(392), 133-194; English transl., Russian Math. Surveys 65 (2010), no. 2, 319-379. MR2668802 (2011j:37107)

[15] B. A. Dubrovin, Theta-functions and nonlinear equations, Uspekhi Mat. Nauk. 36 (1981), no. 2(218), 11-80; English transl., Russian Math. Surveys 36 (1981), no. 2, 11-92. MR616797 (83i:35149)

[16] L. Erdős, Universality of Wigner random matrices: a survey of recent results, Uspekhi Mat. Nauk. 66 (2011), no. 3(399), 67-198; English transl., Russian Math. Surveys 66 (2011), no. 3, 507-626. MR.2859190

[17] B. Eynard, Large $N$ asymptotics of orthogonal polynomials: from integrability to algebraic geometry, Applications of Random Matrices in Physics, 489-513, Springer, Dordrecht, 2006 (NATO Sci. Ser. II: Math. Phys. Chem., 221). MR2232120 (2007h:33005)

[18] G. M. Goluzin, Geometric theory of functions of a complex variable, Second edition, Nauka, Moscow, 1966; English transl., Translations of Mathematical Monographs, vol. 26, Amer. Math. Soc., Providence, RI, 1969. MR 0247039(40:308)

[19] A. A. Gonchar, Rational approximations of analytic functions, Current problems in mathematics. No. 1, 83-106, Ross. Akad. Nauk, Inst. Mat. im. Steklova, Moscow, 2003 (Russian). MR2141823 (2006m:30066)

[20] A. A. Gonchar and E. A. Rakhmanov, On the convergence of simultaneous Padé approximants for systems of functions of Markov type. Number theory, mathematical analysis and their applications. Trudy Mat. Inst. Steklov 157 (1981), 31-48, 234 (Russian). MR651757 (84d:41028)

[21] A. A. Gonchar and E. A. Rakhmanov, The equilibrium measure and distribution of zeros of extremal polynomials, Mat. Sb. 125(167) (1984), no. 1(9), 117-127; English transl., Math. USSR-Sb. 53 (1986), no. 1, 119-130. MR760416 (86f:41002)

[22] A. A. Gonchar and E. A. Rakhmanov, Equilibrium distributions and the rate of rational approximation of analytic functions, Mat. Sb. 134(176) (1987), no. 3(11), 306-352; English transl., Math. USSR-Sb. 62 (1989), no. 2, 305-348. MR922628 (89h:30054)

[23] A. V. Komlov and S. P. Suetin, Widom's formula for the leading coefficient of a polynomial that is orthonormal with respect to a variable weight, Uspekhi Mat. Nauk 67 (2012), no. 1, 183-184; English transl., Russian Math. Surveys 67 (2012), no. 1, 183-185. MR2961473

[24] A. V. Komlov and S. P. Suetin, The Ahiezer-Nuttall asymptotic formula for polynomials orthonormal with respect to a varying weight, 2012. (To appear)

[25] A. B. J. Kuijlaars and K. T.-R. McLaughlin, Generic behavior of the density of states in random matrix theory and equilibrium problems in the presence of real analytic external fields, Comm. Pure Appl. Math. 53 (2000), no. 6, 736-785. MR1744002 (2001f:31003)

[26] A. B. J. Kuijlaars and P. M. J. Tibboel, The asymptotic behaviour of recurrence coefficients for orthogonal polynomials with varying exponential weights, J. Comput. Appl. Math. 233 (2009), no. 3, 775-785. MR2583016 (2010m:42054)

[27] N. S. Landkof, Foundations of modern potential theory, Nauka, Moscow, 1966; English transl., Die Grundlehren der Mathematischen Wissenschaften, Band 180. Springer-Verlag, New YorkHeidelberg, 1972. MR0350027 (50:2520)

[28] A. Martinez-Finkelshtein, E. A. Rakhmanov, and S. P. Suetin, Heine, Hilbert, Padé, Riemann, and Stieltjes: John Nuttall's work 25 years later, Recent Advances in Orthogonal Polynomials, Special Functions, and Their Applications. Providence, RI, Amer. Math. Soc., 2012, 165-193. (Contemporary Math., 578). MR2964145

[29] H. N. Mhaskar and E. B. Saff, Extremal problems for polynomials with exponential weights, Trans. Amer. Math. Soc. 285 (1984), no. 1, 203-234. MR748838(86b:41024)

[30] J. Nuttall, Asymptotics of diagonal Hermite-Padé polynomials, J. Approx. Theory 42 (1984), 299386. MR769985 (86j:41017)

[31] J. Nuttall, Padé polynomial asymptotics from a singular integral equation, Constr. Approx 6 (1990), no. 2, 157-166. MR.1036606 (91e:41017)

[32] J. Nuttall and R. S. Singh, Orthogonal polynomials and Padé approximants associated with a system of arcs, J. Approx. Theory 21 (1977), 1-42. MR0487173 (58:6833)

[33] L. Pastur, From random matrices to quasi-periodic Jacobi matrices via orthogonal polynomials, J. Approx. Theory 139 (2006), no. 1-2, 269-292. MR2220042 (2006m:47057) 
[34] L. Pastur and M. Shcherbina, Universality of the local eigenvalue statistics for a class of unitary invariant random matrix ensembles, J. Statist. Phys. 86 (1997), no. 1-2, 109-147. MR1435193 (98b:82037)

[35] L. Pastur and M. Shcherbina, On the edge universality of the local eigenvalue statistics of matrix models, Mat. Fiz. Anal. Geom. 10 (2003), no. 3, 335-365. MR2012268 (2004g:60012)

[36] E. A. Rakhmanov, The asymptotic behavior of the ratio of orthogonal polynomials, Mat. Sb. 103(145) (1977), no. 2(6), 237-252; English transl., Mat. Sb. (N.S.) 103(145) (1977), no. 2, 237252, 319. MR $0445212(56: 3556)$

[37] E. A. Rakhmanov, The convergence of diagonal Padé approximants, Mat. Sb. 104(146) (1977), no. 2, 271-291; English transl., Math. USSR-Sb. 33 (1977), no. 2, 243-260. MR0492292 (58:11430)

[38] E. A. Rakhmanov, Asymptotic properties of polynomials that are orthogonal on the real axis, Dokl. Akad. Nauk SSSR 261 (1981), no. 2, 282-284 (Russian). MR638916 (83a:42020)

[39] E. A. Rakhmanov, Asymptotic properties of orthogonal polynomials on the real axis, Mat. Sb. 119(161) (1982), no. 2(10), 163-203, 303; English transl., Math. USSR-Sb. 47 (1984), no. 1, 155193. MR675192(84e:42025)

[40] E. B. Saff and V. Totik, Logarithmic potentials with external fields, Appendix B by Thomas Bloom. Grundlehren der Mathematischen Wissenschaften, 316. Springer-Verlag, Berlin, 1997. MR,1485778 (99h:31001)

[41] M. Shcherbina, Central limit theorem for linear eigenvalue statistics of orthogonally invariant matrix models, Zh. Mat. Fiz. Anal. Geom. 4 (2008), no. 1, 171-195, 204. MR2404179(2009b:82043)

[42] S. P. Suetin, On the uniform convergence of diagonal Padé approximants for hyperelliptic functions, Mat. Sb. 191 (2000), no. 9, 81-114; English transl., Sb. Math. 191 (2000), no. 9-10, 1339-1373. MR.1805599(2002e:30034)

[43] S. P. Suetin, On asymptotic properties of poles of diagonal Padé approximants for some generalizations of Markov functions, Mat. Sb. 193 (2002), no. 12, 105-133; English transl., Sb. Math. 193 (2002), no. 11-12, 1837-1866. MR1992106 (2004j:30010)

[44] S. P. Suetin, Comparative asymptotic behavior of solutions and trace formulas for a class of difference equations, Sovremennye Problemy Matematiki [Current Problems in Mathematics], 6, 3-74. MIAN, Moscow, 2006 (Russian). MR2254701(2008m:47044)

[45] G. Springer, Introduction to Riemann surfaces, Addison-Wesley Publishing Company, Inc., Reading, Mass., 1957. MR0092855(19:1169g)

[46] G. Szegő, Orthogonal polynomials, Amer. Math. Soc., Providence, RI, 1959. MR0106295

[47] D. N. Tulyakov, Asymptotics of Plancherel-Rotach type for solutions of linear recurrence relations with rational coefficients, Mat. Sb. 201 (2010), no. 9, 111-158; English transl., Sb. Math. 201 (2010), no. 9-10, 1355-1402. MR2760462 (2012a:39002)

[48] H. Widom, Extremal polynomials associated with a system of curves in the complex plane, Adv. Math. 3 (1969), no. 2, 127-232. MR0239059 (39:418)

[49] E. I. Zverovich, Boundary value problems in the theory of analytic functions in Hölder classes on Riemann surfaces, Uspekhi Mat. Nauk 26 (1971), no. 1, 113-179. MR0409841 (53:13593)

Steklov Mathematical Institute of the Russian Academy of Sciences

E-mail address: komlov@mi.ras.ru

Steklov Mathematical Institute of the Russian Academy of Sciences

E-mail address: suetin@mi.ras.ru 